\title{
Photon Energy-Dependent Hysteresis Effects in Lead Halide Perovskite Materials
}

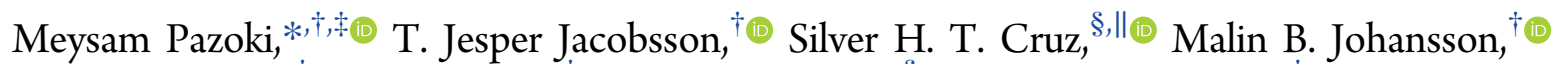 \\ Roghayeh Imani, ${ }^{\dagger}$ Jolla Kullgren, ${ }^{\dagger \odot}$ Anders Hagfeldt, ${ }^{\S}$ Erik M. J. Johansson, ${ }^{\dagger}$ Tomas Edvinsson, ${ }^{\ddagger \oplus}$ \\ and Gerrit Boschloo* ${ }^{\dagger} \dagger$
}

${ }^{\dagger}$ Department of Chemistry, Ångström Laboratory, Uppsala University, Box 538, 75121 Uppsala, Sweden

${ }^{\S}$ Laboratory for Photomolecular Science, Institute of Chemical Sciences and Engineering, École Polytechnique Fédérale de Lausanne, $\mathrm{CH}$-1015-Lausanne, Switzerland

${ }^{*}$ Department of Engineering Sciences-Solid State Physics, Uppsala University, Box 534, 75121 Uppsala, Sweden

"Benemérita Universidad Autónoma de Puebla, CIDS, Avenida San Claudio y 18 Sur, Col. San Manuel, Ciudad Universitaria, CP 72570, P.O. Box 1067, 7200 Puebla, México

\section{Supporting Information}

ABSTRACT: Lead halide perovskites have a range of spectacular properties and interesting phenomena and are a serious candidate for the next generation of photovoltaics with high efficiencies and low fabrication costs. An interesting phenomenon is the anomalous hysteresis often seen in current-voltage scans, which complicates accurate performance measurements but has also been explored to obtain a more comprehensive understanding of the device physics. Herein, we demonstrate a wavelength and illumination intensity dependency of the hysteresis in state-of-the-art perovskite solar cells with $18 \%$ power conversion efficiency (PCE), which gives new insights into ion migration. The perovskite devices show lower hysteresis under illumination with near band edge (red) wavelengths compared to more energetic (blue) excitation. This can be rationalized with thermalization-assisted ion movement or thermalization-assisted vacancy generation. These explanations are supported by the dependency of the photovoltage decay with illumination time and excitation wavelength, as well as by impedance spectroscopy. The suggested mechanism is that high-energy photons create hot charge carriers that either through thermalization can create additional vacancies or by release of more energetic phonons play a role in overcoming the activation energy for ion movement. The excitation wavelength dependency of the hysteresis presented here gives valuable insights into the photophysics of the lead halide perovskite solar cells.

\section{INTRODUCTION}

Lead halide perovskite materials have recently gained popularity in the solar cell research community by showing promising photovoltaic performances ${ }^{1}$ comparable with 40 year old PV technologies like CIGS and CdTe. ${ }^{2,3}$ The lead halide perovskites have spectacular material properties such as a large extinction coefficient in the visible range, ${ }^{4}$ high carrier diffusion lengths, ${ }^{5}$ high charge mobilities, ${ }^{6}$ high dielectric constant, ${ }^{4}$ and suitable band gap and valence/conduction band $(\mathrm{VB} / \mathrm{CB})$ positions for commonly used charge-selective materials. These make them interesting candidates for photovoltaic applications, and they have demonstrated the highest ever seen power conversion efficiency (PCE) increase rate for solar cell materials. ${ }^{7}$ Methylammonium (MA) lead iodide perovskite (MALIP) ${ }^{8,9}$ is the material that has attracted the most interest initially. The highest reported efficiencies have, however, been reported for mixed-ion halide perovskites in which some of the iodide and MA have been replaced with bromide and formamidinium (FA)/caesium, respectively. ${ }^{10,11}$

A hysteresis in the current voltage (IV) measurement is frequently seen in perovskite devices. ${ }^{12,13}$ This is an issue that needs to be understood in more detail. It would not only simplify the measurements of the correct PCE and device characteristics but also pave the way for a deeper understanding of the physics behind the remarkable photovoltaic performance. The hysteresis in MALIP solar cells was initially reported to depend on the scan rate, illumination history, device architecture, and scan direction ${ }^{12,13}$ and has been investigated by several groups. ${ }^{14-24}$ The same type of hysteresis, with varying magnitude, has been reported in perovskites with a

Received: July 10, 2017

Revised: November 3, 2017

Published: November 20, 2017 
wide range of compositions, including the mixed perovskites with record efficiencies.

The observed hysteresis depends on the nature of the chargeselective contacts ${ }^{25}$ and can be reduced in highly efficient devices by different strategies, e.g., changing the thickness of the mesoporous $\mathrm{TiO}_{2}$ layer ${ }^{14}$ or using organic-selective contacts such as fullerene ${ }^{26}$ and PCBM ([6,6]-phenyl C61-butyric acid methyl ester). ${ }^{27}$ Ion migration, ${ }^{18,20}$ interface electric fields, ${ }^{28}$ ferroelectric and polarizability domains, ${ }^{15,19}$ and interface charge accumulation ${ }^{24}$ have all been suggested to be fully or partly responsible for this effect, although a complete and convincing physical picture is still lacking. The hysteresis in current voltage measurements is indicative of phenomena that are evolving in time until a relaxed steady state can be reached. The time scale of these phenomena is comparable to or longer than the delay in between the voltage scan steps, i.e., 10-100 $\mu \mathrm{s}$. Nevertheless, a combination of all these suggestions could be a more comprehensive explanation for the hysteresis as they are interlinked processes. Examples of how they could be related have been discussed in terms of photoinduced ion migration, ${ }^{29}$ ion migration-induced lattice structural changes, ${ }^{30}$ rotation of organic dipole coupled with ion migration, and charge accumulation at interfaces due to ion migration. ${ }^{17}$ Recently, it has been reported that by considering the intrinsic ion migration within perovskites together with interface recombination the hysteresis can be explained in the devices employing both organic and inorganic charge-selective contacts. $^{18}$

In this work, we investigated the hysteresis photon energy dependence to develop a more complete mechanistic picture. We report a distinctive excitation wavelength dependency of the hysteresis in high-efficiency mixed-halide perovskite devices. This gives important insights into the ionic movement and the photophysics in the perovskites. The observed phenomena are explained in detail in terms of phonon-related ion migration and different electronic transitions.

\section{METHODS}

Device Fabrication. As substrate for the devices, FTO NSG 10 was used. The substrates were cleaned in freshly prepared piranha solution composed of five parts concentrated $\mathrm{H}_{2} \mathrm{SO}_{4}$ and two parts $30 \% \mathrm{H}_{2} \mathrm{O}_{2}$. The substrates were soaked in the piranha solution for $10 \mathrm{~min}$ and then rinsed in water and then ethanol. They were thereafter treated in a UV-ozone cleaner for $10 \mathrm{~min}$. A hole bocking layer of $\mathrm{TiO}_{2}$ was deposited on the cleaned FTO substrates using spray pyrolysis (see the Supporting Information for the details). This procedure gives a compact layer of anatase with a thickness of around 20-30 nm. On top of the compact $\mathrm{TiO}_{2}$ layer deposited by spray pyrolysis, a mesoporous scaffold of $\mathrm{TiO}_{2}$ nanoparticles was deposited by spin coating. $\mathrm{TiO}_{2}$ paste (30 NR-D) was bought from Dyesol and was dissolved in ethanol at a concentration of $150 \mathrm{mg} / \mathrm{mL}$. On each substrate $(1.4 \times 2.4 \mathrm{~cm}) 50 \mu \mathrm{L}$ of the $\mathrm{TiO}_{2}$ solution was applied and spin coated at $4000 \mathrm{rpm}$ with an acceleration of $2000 \mathrm{rpm} / \mathrm{s}$ for $10 \mathrm{~s}$. The substrates with mesoporous $\mathrm{TiO}_{2}$ were sintered at $450{ }^{\circ} \mathrm{C}$ in air on a hot plate for $30 \mathrm{~min}$ and then slowly cooled to ambient temperature. Prior to perovskite deposition, the substrates with mesoporous $\mathrm{TiO}_{2}$ underwent a lithium treatment. On the substrates, $100 \mu \mathrm{L}$ of $35 \mathrm{mM}$ lithium bistrifluoromethanesulfonimidate, Li-TFSI, in acetonitrile was applied, and thereafter the substrates were spun at $3000 \mathrm{rpm}$ for $10 \mathrm{~s}$. The substrates were then thermally annealed in air at 450 ${ }^{\circ} \mathrm{C}$ for $30 \mathrm{~min}$ and then slowly cooled down to $150{ }^{\circ} \mathrm{C}$, where after they were brought directly into a glovebox for perovskite deposition. For devices with a $\mathrm{SnO}_{2}$ electron-selective contact, the $\mathrm{SnO}_{2}$ was deposited by atomic layer deposition, ALD, at $120{ }^{\circ} \mathrm{C}$ using a Savanah ALD 100 from Cambridge Nanotech Inc. The substrates with $\mathrm{SnO}_{2}$ were directly prior to perovskite deposition treated in a UV-ozone cleaner for $10 \mathrm{~min}$. The precursor solutions were prepared in a glovebox with nitrogen atmosphere. Stock solutions of $\mathrm{PbI}_{2}$ and $\mathrm{PbBr}_{2}$ were prepared in advance, whereas the final precursor solutions were prepared just before the perovskite deposition. The solvent used for the perovskite solutions was a mixture of anhydrous dimethylformamide, DMF, and anhydrous dimethyl sulfoxide, DMSO, in the proportion $4: 1$ by volume. Four master solutions were prepared: $\mathrm{PbI}_{2}$ and $\mathrm{CH}_{3} \mathrm{NH}_{3} \mathrm{I}$ in DMF/DMSO, $\mathrm{PbI}_{2}$ and $\mathrm{CH}\left(\mathrm{NH}_{2}\right)_{2} \mathrm{I}$ in DMF/DMSO, $\mathrm{PbBr}_{2}$ and $\mathrm{CH}_{3} \mathrm{NH}_{3} \mathrm{Br}$ in DMF/ DMSO, and $\mathrm{PbBr}_{2}$ and $\mathrm{CH}\left(\mathrm{NH}_{2}\right)_{2} \mathrm{Br}$ in DMF/DMSO. These were mixed in the right proportions to get the final precursor solution with the following concentrations: $\left[\mathrm{Pb}^{2+}\right]=1.25 \mathrm{M}$, $\left[\mathrm{PbI}_{2}\right]=1.04 \mathrm{M},\left[\mathrm{PbBr}_{2}\right]=0.21 \mathrm{M},[\mathrm{FAI}]=0.63 \mathrm{M},[\mathrm{MAI}]=$ $0.32 \mathrm{M},[\mathrm{FABr}]=0.13 \mathrm{M},[\mathrm{MABr}]=0.06 \mathrm{M}$. For each sample, $35 \mu \mathrm{L}$ of the precursor solution was spread over the substrate, which thereafter was spin coated using a two-step program. The first step was a spreading step using a rotation speed of 1000 $\mathrm{rpm}$ with an acceleration of $200 \mathrm{rpm} / \mathrm{s} \mathrm{rpm}$ for $10 \mathrm{~s}$. That step is immediately, without pause, followed by the second step where the films were spun at $4000 \mathrm{rpm}$ for $30 \mathrm{~s}$ using an acceleration of $2000 \mathrm{rmp} / \mathrm{s}$. During the second step, when approximately $15 \mathrm{~s}$ of the program remains, $100 \mu \mathrm{L}$ of anhydrous chlorobenzene was applied on the spinning film with a hand-held automatic pipet.

Directly after spin coating, the films were placed on a hot plate at $100{ }^{\circ} \mathrm{C}$, where they were annealed between 30 and 70 min depending on the perovskite composition. At this temperature the transformation into the perovskite is visually seen to occur within the time frame of $1 \mathrm{~min}$. After heat treatment the samples were cooled to ambient temperature where after the solid state hole conductor was spin coated on top of the films. A $70 \mathrm{mM}$ solution of Spiro-MeOTAD (spiro) dissolved in chlorobenzene was used as a hole conductor. To improve the performance of the spiro, three different additives were added: 4-tert-butylpyridine, $1.8 \mathrm{M} \mathrm{Li}$-TFSI in acetonitrile, and $0.25 \mathrm{M} \mathrm{Co}[t-\mathrm{BuPyPz}]_{3}[\mathrm{TFSI}]_{3}$, also known as FK209, in acetonitrile. The Spiro:FK209:Li-Tfsi:TBP molar ratio was 1:0.05:0.5:3.3. The spiro was deposited by spin coating at 4000 rpm for $20 \mathrm{~s}$. A $50 \mu \mathrm{L}$ amount of the solution was deposited on the spinning film using a hand-held automatic pipet a few seconds into the spinning program. The samples were stored in a desiccator pumped under vacuum for 1 day, and the back contact was then deposited. The front and back contact were composed of an $80 \mathrm{~nm}$ thick gold film deposited by physical vapor deposition at a pressure of around $2 \times 10^{-5}$ Torr using an evaporator from Leica, EM MED020.

Device Characterization. All measurements were done in air atmosphere and room temperature; our mixed-perovskite devices were stable, keeping the high efficiency in the interval of measurement; please see Figures S10 and S11 of the Supporting Information for typical stability data for thousands of seconds. The same intensity of incoming light $(30 \mathrm{~mW}$ $\mathrm{cm}^{-2}$ ) or the same photon flux was used for the excitation. The light sources used were a solar simulator (Newport class ABB) for white light and two LED sources: Thorlabs M625L3 for red light $(625 \mathrm{~nm})$ and Thorlabs M470L3 for blue light $(470 \mathrm{~nm})$. A power meter (Thorlabs PM 100D) with a mask $\left(0.1256 \mathrm{~cm}^{2}\right.$ 
area) was used for measuring the intensity of monochromatic light, while a calibrated Si diode was used for measuring the intensity of white light. The implemented mask size for all measurements was $0.1256 \mathrm{~cm}^{2}$.

A minimum of 3-4 devices of $\mathrm{MAPbI}_{3}$ and mixed perovskite were tested. For $I V$ measurement before each forward-reverse scan, the cell was kept in the dark at $0 \mathrm{~V}$ bias for a few minutes. IPCE of the devices were measured with a lab-made setup with a xenon lamp, a monochromator, a Si diode detector connected to a lock-in amplifier, and a computer. Bias light was produced by the above-mentioned light sources for red, blue, and white light, and its intensity was measured by the power meter/silicon diode. A shutter with $30 \mathrm{~Hz}$ frequency and the DC bias light were implemented for ac IPCE, and the result is reported in Figure S1. EIS spectra were recorded by an Autolab potentiostat in open circuit with illumination or with a constant current mode plus illumination. The latter corresponds to a working condition point in the middle of the $I V$ curve. The implemented illumination intensities were calibrated in order to have the same number of incoming photons or the same intensities for blue and red excitations. Frequencies from 1 $\mathrm{MHz}$ to $10 \mathrm{mHz}$ were chosen.

$V_{\text {OC }}$ decay measurements of devices were done initially for red and blue illumination. Directly after those measurements, the device was light soaked in either red or blue light for about $5 \mathrm{~min}$ and then again measured for $V_{\mathrm{OC}}$ decay.

All characterization studies were done in ambient atmosphere with a maximum of $30 \%$ humidity. All of the observed changes in the $I V$ curves were reversible. By keeping the films in the dark for sufficiently long times, the films return to their initial conditions before each measurement. The scan to forward bias $\left(0 \mathrm{~V}\right.$ to open-circuit potential $\left.\left(V_{\mathrm{OC}}\right)\right)$ is the forward scan; the reverse scan is from $V_{\mathrm{OC}}$ to $0 \mathrm{~V}$. More details of experimental methods can be found in the Supporting Information.

\section{RESULTS AND DISCUSSION}

The schematic of the mixed-perovskite devices is presented in Figure 1a. IV measurements were performed on efficient perovskite solar cells under different illumination conditions: simulated sunlight (AM1.5G), blue light, or red light (Figure $1 \mathrm{~b}$ and $1 \mathrm{c}$ ). Since the thermalization losses of the initially created charge carriers are smaller under red light illumination than under both blue and white light illuminations, higher device efficiencies are calculated for red light illumination. For our best device the PCE under red light $(625 \mathrm{~nm})$ was as high as $42 \%$, compared to $19 \%$ under AM $1.5 \mathrm{G}$.

In line with other reports ${ }^{13,16}$ we observed a hysteresis in the $I V$ measurements of the mixed-halide perovskite solar cells. ${ }^{11}$ Interestingly, the $I V$ measurements revealed a clear excitation wavelength dependency: red light illumination did in all cases result in less hysteresis than blue light. The difference in the fill factor (FF) between the forward scan $\left(0 \mathrm{~V} \rightarrow V_{\mathrm{OC}}\right)$ and the reverse scan $\left(V_{\mathrm{OC}} \rightarrow 0 \mathrm{~V}\right)$ was a few up to $20 \%$, while $V_{\mathrm{OC}}$ and short-circuit current density $\left(J_{\mathrm{SC}}\right)$ did not show significant changes. We will quantify the hysteresis in $I V$ curves by defining a hysteresis factor $(H F)$ as follows

$$
\mathrm{HF}_{\mathrm{FF}}(\%)=\left|\frac{\mathrm{FF}(\text { forward })-\mathrm{FF}(\text { backward })}{\mathrm{FF}(\text { backward })}\right| \times 100
$$

A similar trend was observed in the hysteresis for the MALIP devices (see SI). Statistics of the hysteresis and device
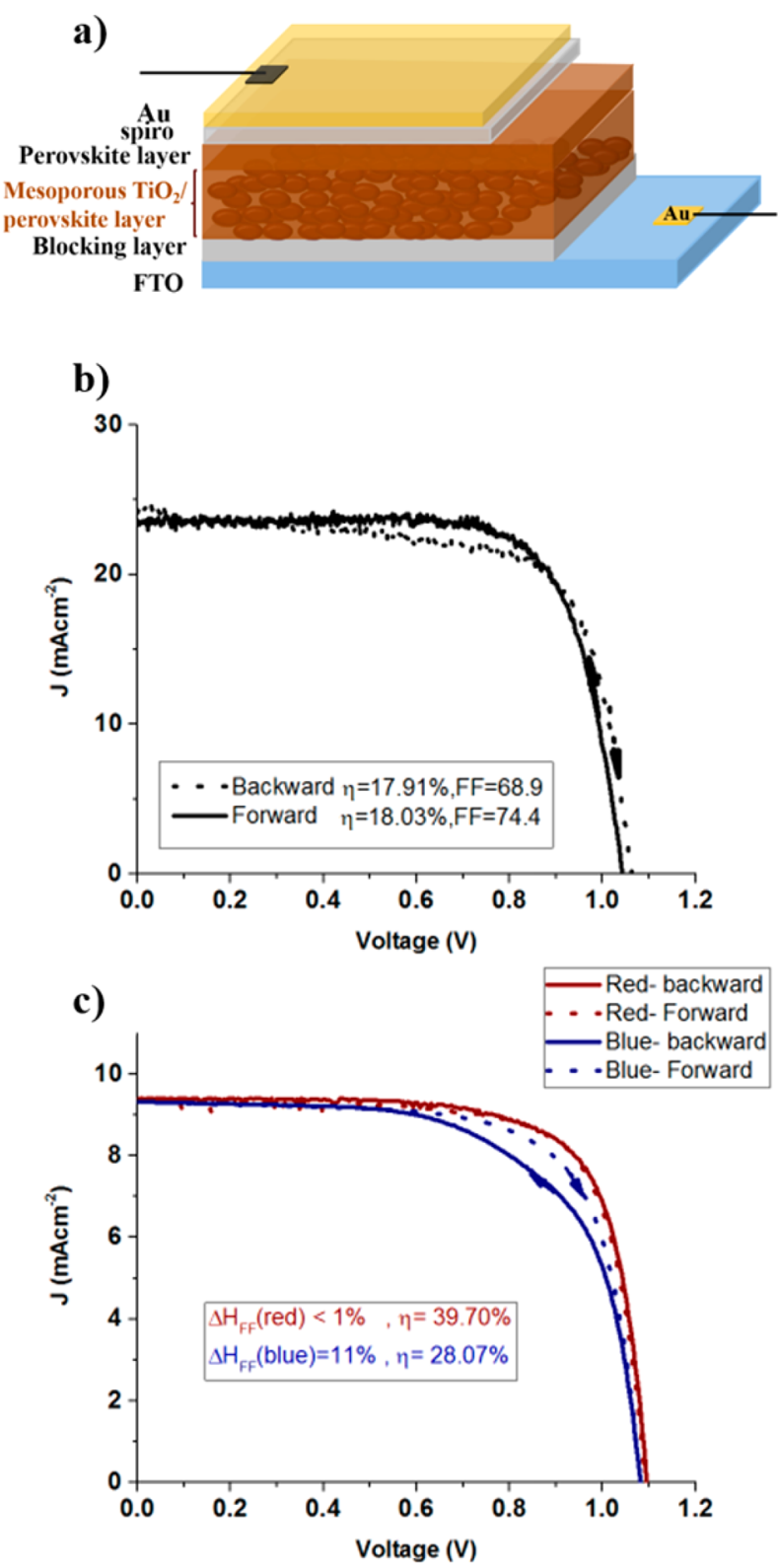

Figure 1. (a) Schematic of the mixed-perovskite device. Typical forward $\left(0 \mathrm{~V} \rightarrow V_{\mathrm{OC}}\right)$ and reverse $\left(V_{\mathrm{OC}} \rightarrow 0 \mathrm{~V}\right)$ current-voltage scans for mixed-halide perovskite $\left(\mathrm{MA}_{0.33} \mathrm{FA}_{0.66} \mathrm{PbI}_{2.5} \mathrm{Br}_{0.5}\right)$ devices illuminated by different light sources of (b) white light $\left(100 \mathrm{~mW} \mathrm{~cm}^{-2}\right)$ and (c) red $\left(625 \mathrm{~nm}, 35 \mathrm{~mW} \mathrm{~cm}^{-2}\right)$ or blue light $\left(470 \mathrm{~nm}, 47 \mathrm{~mW} \mathrm{~cm}^{-2}\right)$, yielding the same incident photon flux. Scan rate is $10 \mathrm{mV} \mathrm{s}^{-1}(\mathrm{~b})$ and $100 \mathrm{mV} \mathrm{s}^{-1}$ (c). Details of the $I V$ curves are reported in the inset.

performance for all measured devices are reported in the SI. In the mixed-perovskite devices, an interplay of the recently reported inverted ${ }^{31}$ and normal hysteresis can be found at different light intensities or scan rates, ${ }^{31}$ which was observed in a few of the devices (S5, Supporting Information). For the typical inverted hysteresis a larger $\mathrm{FF}$ and $V_{\mathrm{OC}}$ are observed for the forward scans due to the presence of extraction barriers at the interfaces (for mixed perovskites). This is in contrast to the case for the normal hysteresis, ${ }^{16}$ which will give a higher FF and $V_{\text {OC }}$ for the reverse scans, Table 1 .

Special care was taken to keep all devices at the same initial condition with respect to illumination and bias before measurement to eliminate the effect of illumination and bias 
Table 1. Fill Factor from Forward $\left(\mathrm{FF}_{\mathrm{f}}\right)$ and Reverse $\left(\mathrm{FF}_{\mathrm{r}}\right)$ Scans and Hysteresis Factor (HF) for Two Representative Devices of Different Compositions $\left(\mathrm{FA}_{0.67} \mathrm{MA}_{0.33} \mathrm{PbI}_{2.5} \mathrm{Br}_{0.5}\right.$ and MALIP) ${ }^{a}$

\begin{tabular}{lllllll}
\hline & \multicolumn{3}{c}{ Mixed Perovskite } & \multicolumn{3}{c}{ MAPbl $_{3}$ Perovskite } \\
\hline & $\mathrm{FF}_{\mathrm{f}}$ & $\mathrm{FF}_{\mathrm{r}}$ & $\mathrm{HF} / \%$ & $\mathrm{FF}_{\mathrm{f}}$ & $\mathrm{FF}_{\mathrm{r}}$ & $\mathrm{HF} / \%$ \\
\hline $\begin{array}{l}\text { Red } \\
(625 \mathrm{~nm})\end{array}$ & 0.73 & 0.73 & $<1$ & 0.54 & 0.72 & 24 \\
\hline $\begin{array}{l}\text { Blue } \\
(470 \mathrm{~nm})\end{array}$ & 0.71 & 0.64 & 11 & 0.44 & 0.65 & 28 \\
\hline
\end{tabular}

${ }^{a_{T}}$ The scan rate was $10 \mathrm{mV} \mathrm{s}^{-1}$. For statistics on all measured devices, see SI.

history (see SI for details). Under open-circuit illumination conditions, accumulation of electrons at the $\mathrm{FTO} / \mathrm{TiO}_{2}$ contact and holes at the spiro/Au contacts will take place. Mobile ions and ion vacancies in the perovskite layer will move in the resulting electric field. Specifically, positively charged iodide vacancies, believed to be the dominating ionic defect, will move toward $\mathrm{FTO} / \mathrm{TiO}_{2}$ and away from spiro/Au contact under these conditions. At $0 \mathrm{~V}$ (short circuit), on the other hand, no charge accumulation takes place at the contacts and the internal electric field will be lower as a result. Ionic defects will be closer to their equilibrium position in the bulk of the perovskite film. The observed hysteresis has been explained by a difference in the profile of ionic species within the perovskite film at reverse/ forward scans, which can affect charge extraction at the contact and recombination. Therefore, one expects that a higher degree of ion displacement can cause a stronger hysteresis.

Herein, devices with both $\mathrm{TiO}_{2}$ and $\mathrm{SnO}_{2}$ electron blocking layers have been investigated, i.e., cells with the stack sequences of $\mathrm{FTO} / \mathrm{TiO}_{2} /$ mesoporous $\mathrm{TiO}_{2} /$ perovskite/spiro/Au and $\mathrm{FTO} / \mathrm{SnO}_{2} /$ perovskite/spiro/Au. $\mathrm{SnO}_{2}$ blocking layer is used since it has been reported to reduce the inverted hysteresis in mixed-perovskite devices in planar configuration ${ }^{32}$ wherefore a comparison between devices with $\mathrm{TiO}_{2}$ and $\mathrm{SnO}_{2}$ electron transport layers (ETL) could provide additional information. The trend in hysteresis with respect to excitation wavelength was the same for the devices with $\mathrm{TiO}_{2}$ and $\mathrm{SnO}_{2}$ ETL. The excitation wavelength dependency of the hysteresis can thus not be explained merely by recombination rate differences at the contact to the ETL as has been suggested ${ }^{31}$ but must at least in part be linked to intrinsic effects in the lead halide perovskite (S5 of SI).

To further investigate this behavior, IV measurements were conducted under different light intensities for different excitation wavelengths (Figure 2 and SI). While hysteresis is negligible for red light excitation at all intensities, there is clearly less hysteresis at lower intensities for blue light (Figures S3-8, Tables S1-4, section S5, Supporting Information).

The fundamental dissimilarity between $I V$ curves measured under red and blue illumination is also presented for MALIP devices, which do not have inverted hysteresis, even though the magnitude was smaller than for the mixed perovskites (Figure S2, Supporting Information). Here we will primarily focus on the high-efficiency mixed-perovskite devices. Albeit the red illumination gives little or no hysteresis at all light intensities (Figure 2), blue illumination results in a hysteresis that increases with higher light intensities. This wavelengthdependent hysteresis can thus not be explained by different absorption profiles of red and blue photons as previously reported for mixed perovskites. ${ }^{16}$ In the $I V$ curves, a (a)

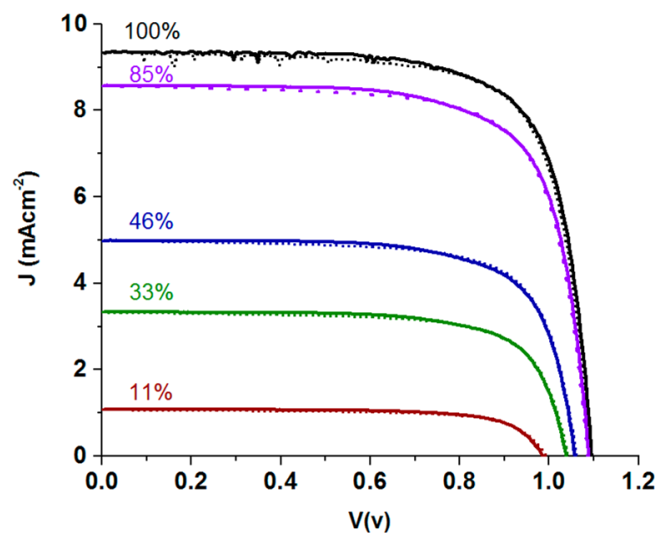

(b)

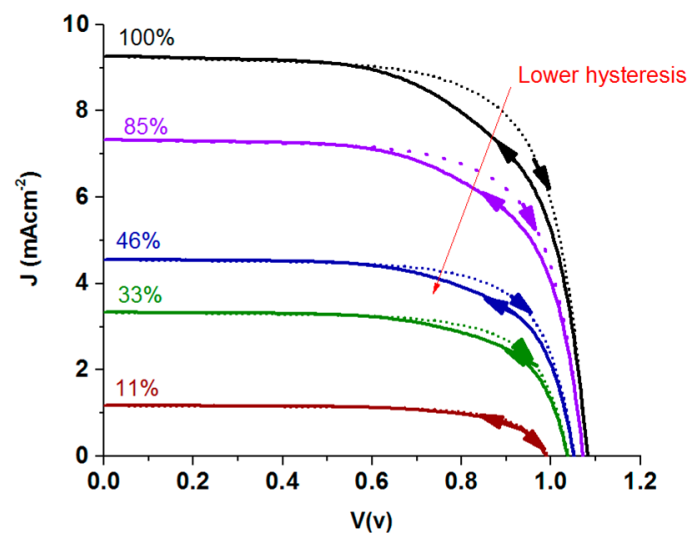

Figure 2. Light intensity-dependent hysteresis in mixed-perovskite device using red (a) and blue (b) light and $100 \mathrm{mV} \mathrm{s}^{-1}$ scan rate. Hysteresis factor is reported in Table S4. Device has a $\mathrm{TiO}_{2}$ ETL with a mesoporous $\mathrm{TiO}_{2}$ film.

competition between the normal and the inverted hysteresis can be observed; however, a more direct and fundamental comparison can be made through impedance spectroscopy and voltage decay measurements under blue and red illuminations.

A correlation between the current decay ${ }^{20,33}$ or voltage decay ${ }^{18,34}$ time constants to the activation energy of the mobile ionic species and ionic-movement-coupled free charge recombination in MALIP has been reported previously. To explore the hysteresis effect in more detail, open-circuit voltage decay measurements were performed under different illumination conditions (Figure 3 and Figure S4, Supporting Information). Before any light soaking and current-voltage measurements on the devices, the voltage rise and decay were relatively rapid, see Figure 3a. Specifically, there was a certain amount of time needed for the voltage to reach a plateau, but there was no long-lived voltage decay. The corresponding rise and decay times are related to the charge recombination in the perovskite film, which are in the microsecond time scale. ${ }^{34}$ Initially, the difference between red and blue illumination was minimal, even under light soaking (see S3 of Supporting Information), while after voltage current measurements a substantial difference was observed (Figure $3 \mathrm{~b}$ and S4 and S5). After forward and reverse $I V$ scans, a long-lived tail in the $V_{\mathrm{OC}}$ decay was found, for which the half time of the decay increased with increased illumination time and excitation energy. Longer illumination times lead to longer tails, and blue light leads to longer tails than red light (Figure 3b). Furthermore, by keeping 

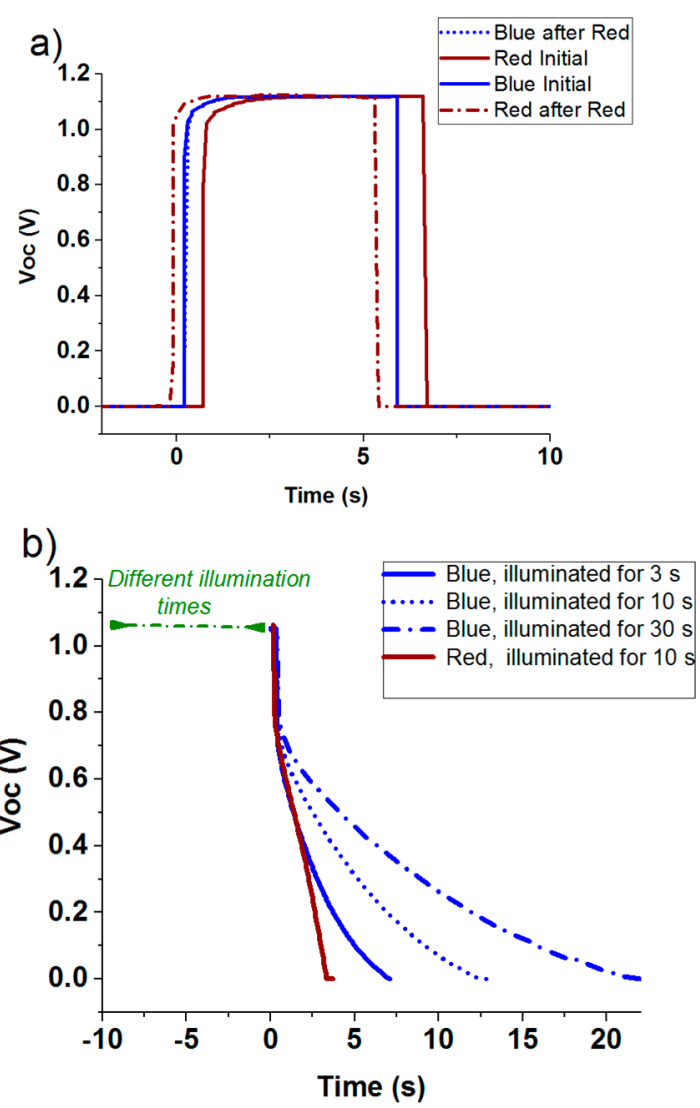

Figure 3. $V_{\mathrm{OC}}$ rise and decay measurements for a mixed-perovskite device after excitation by blue or red light. (a) Red or blue excitation after initial light soaking with red light. (b) $V_{\mathrm{OC}}$ decay measurements performed after $I V$ measurements; device is illuminated during different illumination times mentioned in the legend. Illumination switched off at $1 \mathrm{~s}$. Resulting voltage decay in the dark is shown.

the cell under red illumination after a long time of blue illumination, the decay tails were elongated, higher than for redonly illumination but lower than for only blue illumination.

Also, in impedance spectroscopy (EIS) a significant effect of illumination wavelength was found (Figure 4). The EIS spectra at open circuit show two distinct semicircles in the Nyquist plot (Figure S9) that can be ascribed to electron-hole recombination and ionic transport for the high- and low-frequency semicircle, respectively. ${ }^{34}$ In the Bode plot of the imaginary part of the impedance $\left(z^{\prime \prime}\right)$ vs frequency (Figure 4) two distinct maxima are found for $-z^{\prime \prime}$ which can be used to determine the time constants of the different processes. While the highfrequency time constant is essentially unchanged, the lowfrequency time constant became longer when changing from red to blue light excitation, see Figures 4 and S9. The highfrequency (free carrier recombination) time constant is expected to depend on the number of incoming photons in which a higher number of photons results in a higher recombination rate and consequently a lower impedance in the high-frequency semicircle. The free charge carrier concentration changes versus time $(\mathrm{d} n / \mathrm{d} t)$ is a second-order power function of the carrier density (eq 2)

$$
\frac{\mathrm{d} n}{\mathrm{~d} t}(n) \propto n^{2}
$$

In $V_{\mathrm{OC}}$ decay curves (Figure 3 ), the ionic species may have similar diffusion coefficients; however, the distribution of ions

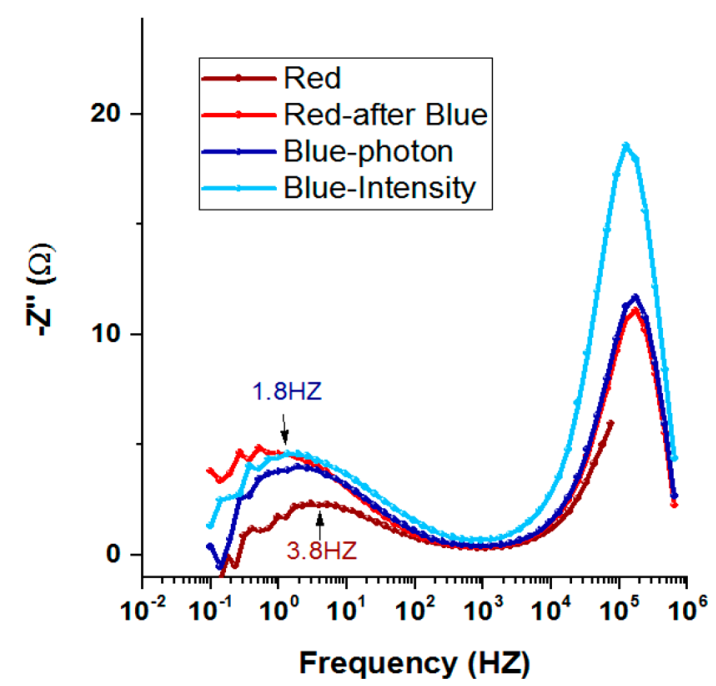

Figure 4. Impedance spectra of a mixed-perovskite $\left(\mathrm{MA}_{0.33} \mathrm{FA}_{0.66} \mathrm{PbI}_{2.5} \mathrm{Br}_{0.5}\right)$ device under blue or red illumination with different intensities at open circuit. Blue-intensity and Blue-photon labels correspond to blue light with exactly the same intensity and the same photon fluxes as red light, respectively.

in the film would be different which can affect the recombination rate within the film. ${ }^{34}$ Therefore, the charge density in eq 2 is different at different locations of the film and depend on the distribution of the ionic species. On the other hand, the maximum of the low-frequency semicircle occurs at lower frequencies for blue illumination than for red. This is likely to be linked to the slower voltage decay for this light color in Figure $3 \mathrm{~b}$. It is worth mentioning that stability issues should be taken into account for these measurements. However, our highly efficient devices were completely stable in the time scales of our presented measurements (see Figures S10 and S11, Supporting Information).

Long illumination times and specifically high-energy photons seems to cause an accumulation of the ionic species at the interfaces under open-circuit conditions. Blue light, more than red light, seems to promote the accumulation of ions in lead halide perovskites. This can be due to a photoinduced effect on the ionic motion, i.e., the activation of ion transport, or due to the creation of additional ionic defects. In the dark, however, ion diffusion will be the same. Carrier motion and recombination will be linked to the ionic motion. The slower voltage decay after blue illumination could come from the strong ion accumulation at the interfaces, leading to a higher capacitance $C$ of the device. The discharge time $R C$ is therefore longer, since $R$ (linked to ion motion) is the same.

Since red and blue illumination have different absorption profiles in the perovskite film, the distribution of ions in the film would be different, which can affect the recombination rate within the film. ${ }^{34}$ Therefore, the charge density in eq 2 is different at different locations of the film and depend on the distribution of the ionic species. This can correlate to more hysteresis in $I V$ scans and lower FF of the device under blue illumination. This wavelength dependency together with the time scale/frequency of the features correlates well to the trend of observed hysteresis effect in the $I V$ scans. The data show a clear difference between the ionic movement resistance under blue and red excitation. Therefore, the difference between the red and the blue illumination is related to not only the recombination profile differences but also the ionic transport. 
Possible Explanations and Suggestions. Ion migration is currently seen as the most plausible explanation for the observed anomalous hysteresis in the perovskite solar cells. Theoretical simulations support this picture. Our measurements give additional insight into the ionic movement hypothesis. In particular, they demonstrate that the hysteresis depends on the excitation wavelength and that it increases with more intense illumination or by longer illumination times. This means that unless the ion movement has reached an equilibrium, a higher photon flux or more energetic photons increases the ionic movement which magnifies the observed difference between the forward and the reverse $I V$ scans. The fundamental difference between absorption of blue and red light is the electron thermalization and cooling energies. The more energetic blue light excites upon absorption the electrons to higher electronic levels, and when the excited electrons relax back to the conduction band edge, they release more phonons. Light-induced structural changes ${ }^{35}$ and photoinduced ion migration have been reported and explained by iodide migration. ${ }^{29}$ Any light-induced or nonlight-induced ion migration will be assisted by vibrational modes of the lattice. Excitation by blue light would lead to a larger number of phonons or might result in excitation of higher vibrational modes that to a greater extent could assist in ionic diffusion compared to the situation of the red light excitation.

A simple transition-state model for the thermal activation of the iodide movement via the jump rate $\Gamma^{35}$ has been widely used to describe the ionic displacement within lead iodide perovskite, where $\nu_{0}$ is the attempt frequency and the $\Delta E$ activation energy

$$
\Gamma=\nu_{\mathrm{o}} \exp (-\Delta E / k T)
$$

A higher number of phonons and possibly also different vibrational modes excited under blue illumination can increase the jump rate and consequently the diffusivity of I vacancies, ${ }^{35}$ which is in line with our experimental data.

Moreover, the reported hysteresis factors would depend on the time scale of the measurement. Slow scan rates that are comparable to the ion migration equilibrium times would lead to a stable steady state charge accumulation in the interfaces between the perovskite and the charge-selective contacts, which would decrease the observed hysteresis. What we see here is that longer illumination times, up to minutes, lead to more ion migration and/or faster ion migration which correlates with higher hysteresis and longer $V_{\mathrm{OC}}$ decay times. In summary, under illumination, ions will move in the perovskite structure which will cause some hysteresis, but for more energetic illumination, the excess phonons generated will speed up this process and increase the observed hysteresis.

Higher Fill Factor for Red Illumination. Electronic transport in the lead halide perovskite materials have with reasonable success been described by the Drude model ${ }^{36}$ in which the mobility $(\mu)$ depends on the scattering time $(\tau)$, the effective mass of charge carriers $\left(m^{*}\right)$, and the electron charge (e) (eq 4)

$$
\mu=\frac{e \tau}{m^{*}}
$$

The electron phonon scattering rather than impurity scattering is the main phenomenon responsible for the observed electron scattering life times in terahertz measurements ${ }^{37}$ and is thus the relevant scattering time in the Drude description.
The higher thermalization for blue-light-created charge carriers implies production of more phonons or that created phonons are more energetic as well as an increase in the temperature of the lattice. A temperature dependence of the mobility of the MALIP has been reported ${ }^{36}$ in which the electron mobility follows the Drude model behavior with a $T^{-3 / 2}$ power law. A $15{ }^{\circ} \mathrm{C}$ increase in the lattice temperature results in a $25 \%$ decrease in the electron mobility at room temperature. That can, at least partially, be responsible for the better FF under the red light excitation. ${ }^{36}$ However, the presented $I V$ curves are in disagreement with reported trends for MALIP device performances at different temperatures, i.e., an increase in FF at expected lower temperature red illumination cannot be observed here, and the FF differences are not temperature related by itself. ${ }^{38}$

The different absorption profiles of red and blue photons can play a role but cannot fully explain the higher fill factors observed under red illumination. Although the presented results are compared for the same number of absorbed photons, the clear light intensity dependency of hysteresis in mixed perovskites and the trends of normal hysteresis in MALIP devices cannot be explained with different absorption profiles (S5 of the Supporting Information as well as the differences in Figures 3 and 4). For example, blue illumination of lower intensity than red illumination still leads to more hysteresis (see SI). A very similar recombination lifetime and different ionic transport lifetime values for red and blue illumination (Figure 4) are further confirming our conclusion concerning photon-assisted ionic movement.

Moreover, for the same illumination wavelength the change in hysteresis with light intensity is in agreement with the idea of thermalization-assisted ion movement. The IPCE of the devices does not show any prominent effect on the bias light wavelength (Figure S1). However, the exact amount of the device temperature changes due to extra thermalization for blue-excited electrons, and its exact contribution to device fill factor is not clear. Furthermore, any mobility change due to thermalization should be unfeasible in both the forward and the reverse scans and therefore has no direct contribution to the observed hysteresis differences between blue and red excitations.

Electron-Phonon Scattering. The more fundamental difference between blue and red excitations can be considered in terms of electron-phonon scattering. Electron-phonon interactions are mainly through the longitudinal optical (LO) phonons which mainly vibrate the $\mathrm{Pb}-\mathrm{I}$ bonds. ${ }^{37} \mathrm{~A}$ possible explanation could be assisted ion (iodide) migration by the phonons that recently has been suggested from device and Stark spectroscopy data. ${ }^{35}$ Regarding the red illumination, it has been suggested that the coupling of near band edge electronic density of states with phonons is different from higher energy levels that makes the cooling lifetime of hot carriers several orders of magnitude longer for the near-band edge illumination. ${ }^{39}$ This is relevant to the experimentally observed longlived hot holes in the MALIP, ${ }^{40,41}$ which is consistent with our observed FF enhancement for red excitations and our suggested phonon-assisted electronic transport herein which is related to the ionic movement.

Possibility of Different Electronic Transitions. According to electronic density of states calculations, the destination energy levels of excited electrons would be different if they are excited by blue or red light. ${ }^{35}$ It has been suggested that a possible electron transfer to the organic cation can occur during 
blue excitation, which can affect the photoinduced ionic movement or any other possible responsible phenomenon for the hysteresis effect. In addition, the possibility of photoinduced traps and their relation to electronic and ionic transport as well as the impacts of more energetic blue photons cannot be excluded. McGehee and coauthors reported photoinduced traps created by blue light illumination within the mixed-perovskite films that is dependent on the illumination light intensity. ${ }^{42}$ Therefore, it is possible that blue illumination creates new vacancies that can give rise to higher ionic conduction and an increase in the hysteresis within the device. The nature and mechanism of the creation of these traps is unclear and needs to be described in terms of how the blue light affects the perovskite structure, such as creation of vacancies and distortions from ion migration. ${ }^{43}$ Recent experimental data for light intensity dependence photoinduced ionic movement ${ }^{43}$ are in line with our suggested explanation for hysteresis data, and on the other hand, the difference in the Stark effect dynamics ${ }^{35}$ in lead halide perovskite films under blue and red light is also a clue to the mechanism of trap creation via structural deformations and ion migration that would depend on different parameters that we shortly discussed here.

\section{CONCLUSIONS}

The frequently observed hysteresis in the $I V$ measurements of lead halide perovskite solar cells is found to be dependent on the wavelength of the illumination, where blue light gives more hysteresis than red. In open-circuit voltage decay measurements after blue light illumination, much slower decay is found compared to after red light. These observations point to the effects of photoinduced ionic conductivity: blue light promotes either ion transport by the electron phonon interactions or by the generation of additional vacancies. Further detailed investigations are needed to pinpoint the precise nature of the effects described here.

\section{ASSOCIATED CONTENT}

\section{S Supporting Information}

The Supporting Information is available free of charge on the ACS Publications website at DOI: 10.1021/acs.jpcc.7b06775.

Materials and methods summary, IPCE of the devices with different bias light, hysteresis behavior of $\mathrm{MAPbI}_{3}$ devices under different wavelength illumination, EIS spectra of devices under red/blue illumination and illumination time hysteresis behavior of mixed-perovskite devices, and corresponding representative table for statistic $s$ of the measured devices (PDF)

\section{AUTHOR INFORMATION}

\section{Corresponding Authors}

*E-mail: meysam.pazoki@kemi.uu.se.

*E-mail: gerrit.boschloo@kemi.uu.se.

\section{ORCID}

Meysam Pazoki: 0000-0001-6776-5460

T. Jesper Jacobsson: 0000-0002-4317-2879

Silver H. T. Cruz: 0000-0003-3191-6188

Malin B. Johansson: 0000-0003-2046-1229

Jolla Kullgren: 0000-0003-3570-0050

Erik M. J. Johansson: 0000-0001-9358-8277

Tomas Edvinsson: 0000-0003-2759-7356
Gerrit Boschloo: 0000-0002-8249-1469

\section{Author Contributions}

M.P. initiated the research, characterized the devices, analyzed the data, and wrote the draft. M.J. and R.I. contributed to the characterization of the devices. J.J. and S.C. fabricated the highefficiency devices. All authors participated in discussion and amended the manuscript. G.B., E.J., and T.E supervised the research.

\section{Notes}

The authors declare no competing financial interest.

\section{ACKNOWLEDGMENTS}

M.P. acknowledges Eva Unger for her kind comments on the manuscript. M.P. and J.K. thank the Åforsk Foundation. Swedish energy agency project number 43294-1 and the StandUP for Energy program are acknowledged for funding support.

\section{REFERENCES}

(1) http://www.nrel.gov/ncpv/images/efficiency_chart.jpg; National Cent. Photovoltaics, 2016.

(2) Etgar, L.; Gao, P.; Xue, Z.; Peng, Q.; Chandiran, A. K.; Liu, B. Mesoscopic $\mathrm{CH}_{3} \mathrm{NH}_{3} \mathrm{PbI}_{3} / \mathrm{TiO}_{2}$ Heterojunction Solar Cells. J. Am. Chem. Soc. 2012, 134, 17396-17399.

(3) Grätzel, M. The Light and Shade of Perovskite Solar Cells. Nat. Mater. 2014, 13, 838-842.

(4) Green, M. A.; Ho-Baillie, A.; Snaith, H. J. The Emergence of Perovskite Solar Cells. Nat. Nat. Photonics 2014, 8, 506-514.

(5) Stranks, S. D.; Stranks, S. D.; Eperon, G. E.; Grancini, G.; Menelaou, C.; Alcocer, M. J. P.; Leijtens, T.; Herz, L. M.; Petrozza, A.; Snaith, H. J. Electron-Hole Diffusion Lengths Exceeding 1 Micrometer in an Organometal Trihalide Perovskite Absorber. Science 2013, 342, $341-344$

(6) Wehrenfennig, C.; Eperon, G. E.; Johnston, M. B.; Snaith, H. J.; Herz, L. M. High Charge Carrier Mobilities and Lifetimes in Organolead Trihalide Perovskites. Adv. Mater. 2014, 26, 1584-1589.

(7) Zhang, W.; Eperon, G. E.; Snaith, H. J. Metal Halide Perovskites for Energy Applications. Nat. Energy 2016, 1, 16048.

(8) Kim, H.-S.; Lee, C. R.; Im, J. H.; Lee, K. B.; Moehl, T.; Marchioro, A.; Moon, S. J.; Humphry Baker, R.; Yum, J. H.; Moser, J. E.; Grätzel, M; Park, N.M. Lead Iodide Perovskite Sensitized All-SolidState Submicron Thin Film Mesoscopic Solar Cell with Efficiency Exceeding 9\%. Sci. Rep. 2012, 2, 591-597.

(9) Lee, M. M.; Teuscher, J.; Miyasaka, T.; Murakami, T. N.; Snaith, H. J. Efficient Hybrid Solar Cells Based on Meso-Superstructured Organometal Halide Perovskites. Science 2012, 338, 643-647.

(10) Jeon, N. J.; Noh, J. H.; Yang, W. S.; Kim, Y. C.; Ryu, S.; Seo, J.; Seok, S. Il. Compositional Engineering of Perovskite Materials for High-Performance Solar Cells. Nature 2015, 517, 476-480.

(11) Jesper Jacobsson, T.; Correa Baena, J. P.; Pazoki, M.; Saliba, M.; Schenk, K.; Grätzel, M.; Hagfeldt, A. Exploration of the Compositional Space for Mixed Lead Halogen Perovskites for High Efficiency Devices. Energy Environ. Sci. 2016, 9, 1706-1724.

(12) Snaith, H. J.; Abate, A.; Ball, J. M.; Eperon, G. E.; Leijtens, T.; Noel, N. K.; Stranks, S. D.; Wang, J. T.; Wojciechowski, K.; Zhang, W. Anomalous Hysteresis in Perovskite Solar Cells. J. Phys. Chem. Lett. 2014, 5, 1511-1515.

(13) Unger, E. L.; Hoke, E. T.; Bailie, C. D.; Nguyen, W. H.; Bowring, A. R.; Heumuller, T.; Christoforo, M. G.; McGehee, M. D. Hysteresis and Transient Behavior in Current-Voltage Measurements of Hybrid-Perovskite Absorber Solar Cells. Energy Environ. Sci. 2014, 7, 3690-3698

(14) Kim, H.; Park, N. Parameters Affecting I-V Hysteresis of $\mathrm{CH}_{3} \mathrm{NH}_{3} \mathrm{PbI}_{3}$ Perovskite Solar Cells: Effects of Perovskite Crystal Size and Mesoporous $\mathrm{TiO}_{2}$ Layer. J. Phys. Chem. Lett. 2014, 5, 2927-2934. 
(15) Wei, J.; Zhao, Y.; Li, H.; Li, G.; Pan, J.; Xu, D.; Zhao, Q.; Yu, D. Hysteresis Analysis Based on the Ferroelectric Effect in Hybrid Perovskite Solar Cells. J. Phys. Chem. Lett. 2014, 5, 3937-3945.

(16) Tress, W.; Marinova, N.; Moehl, T.; Zakeeruddin, S. M.; Mohammad K, N.; Grätzel, M.; Nazeeruddin, M. K.; Grätzel, M. Understanding the Rate-Dependent $\mathrm{J}-\mathrm{V}$ Hysteresis, Slow Time Component, and Aging in $\mathrm{CH}_{3} \mathrm{NH}_{3} \mathrm{PbI}_{3}$ Perovskite Solar Cells: The Role of a Compensated Electric Field. Energy Environ. Sci. 2015, 8, 995-1004.

(17) Mosconi, E.; De Angelis, F. Mobile Ions in Organohalide Perovskites: Interplay of Electronic Structure and Dynamics. ACS Energy Lett. 2016, 1, 182-188.

(18) Calado, P.; Telford, A. M.; Bryant, D.; Li, X.; Nelson, J.; O’Regan, B. C.; Barnes, P. R. F. Evidence for Ion Migration in Hybrid Perovskite Solar Cells with Minimal Hysteresis. Nat. Commun. 2016, 7, 13831.

(19) Frost, J. M.; Butler, K. T.; Walsh, A. Molecular Ferroelectric Contributions to Anomalous Hysteresis in Hybrid Perovskite Solar Cells. APL Mater. 2014, 2, 081506.

(20) Eames, C.; Frost, J. M.; Barnes, P. R. F.; O’Regan, B. C.; Walsh, A.; Islam, M. S. Ionic Transport in Hybrid Lead Iodide Perovskite Solar Cells. Nat. Commun. 2015, 6, 7497.

(21) Ono, L. K.; Raga, S. R.; Wang, S.; Kato, Y.; Qi, Y. TemperatureDependent Hysteresis Effects in Perovskite-Based Solar Cells. J. Mater. Chem. A 2015, 3, 9074-9080.

(22) Yu, H.; Lu, H.; Xie, F.; Zhou, S.; Zhao, N. Native DefectInduced Hysteresis Behavior in Organolead Iodide Perovskite Solar Cells. Adv. Funct. Mater. 2016, 26, 1411-1419.

(23) Miyano, K.; Yanagida, M.; Tripathi, N.; Shirai, Y. Hysteresis, Stability, and Ion Migration in Lead-Halide Perovskite Photovoltaics. J. Phys. Chem. Lett. 2016, 7, 2240-2245.

(24) Chen, B.; Yang, M.; Zheng, X.; Wu, C.; Li, W.; Yan, Y.; Bisquert, J.; Garcia-Belmonte, G.; Zhu, K.; Priya, S. Impact of Capacitive Effect and Ion Migration on the Hysteretic Behavior of Perovskite Solar Cells. J. Phys. Chem. Lett. 2015, 6, 4693-4700.

(25) Kim, H. S.; Jang, I. H.; Ahn, N.; Choi, M.; Guerrero, A.; Bisquert, J.; Park, N. G. Control of I-V Hysteresis in $\mathrm{CH}_{3} \mathrm{NH}_{3} \mathrm{PbI}_{3}$ Perovskite Solar Cell. J. Phys. Chem. Lett. 2015, 6, 4633-4639.

(26) Xu, J.; Buin, A.; Ip, A. H.; Li, W.; Voznyy, O.; Comin, R.; Yuan, M.; Jeon, S.; Ning, Z.; McDowell, J. J. Perovskite-Fullerene Hybrid Materials Suppress Hysteresis in Planar Diodes. Nat. Commun. 2015, 6, 7081 .

(27) Heo, J. H.; Han, H. J.; Kim, D.; Ahn, T. K.; Im, S. H. HysteresisLess Inverted $\mathrm{CH}_{3} \mathrm{NH}_{3} \mathrm{PbI}_{3}$ Planar Perovskite Hybrid Solar Cells with 18.1\% Power Conversion Efficiency. Energy Environ. Sci. 2015, 8, $1602-1608$

(28) Wu, B.; Fu, K.; Yantara, N.; Xing, G.; Sun, S.; Sum, T. C.; Mathews, N. Charge Accumulation and Hysteresis in Perovskite-Based Solar Cells: An Electro-Optical Analysis. Adv. Energy Mater. 2015, 5, 1500829.

(29) deQuilettes, D. W.; Zhang, W.; Burlakov, V. M.; Graham, D. J.; Leijtens, T.; Osherov, A.; Snaith, H. J.; Ginger, D. S.; Stranks, S. D. Photo-Induced Halide Redistribution in Organic-inorganic Perovskite Films. Nat. Commun. 2016, 7, 11683.

(30) Gottesman, R.; Gouda, L.; Kalanoor, B. S.; Haltzi, E.; Tirosh, S.; Rosh-Hodesh, E.; Tischler, Y.; Zaban, A.; Quarti, C.; Mosconi, E. Photoinduced Reversible Structural Transformations in Free-Standing $\mathrm{CH}_{3} \mathrm{NH}_{3} \mathrm{PbI}_{3}$ Perovskite Films. J. Phys. Chem. Lett. 2015, 6, 23322338.

(31) Tress, W. Inverted Current-Voltage Hysteresis in Mixed Perovskite Solar Cells: Polarization, Energy Barriers, and Defect Recombination. Adv. Energy Mater. 2016, 6, 1600396.

(32) Correa Baena, J. P.; Steier, L.; Tress, W.; Saliba, M.; Neutzner, S.; Matsui, T.; Giordano, F.; Jacobsson, T. J.; Kandada, A. R. S.; Zakeeruddin, S. M. Highly Efficient Planar Perovskite Solar Cells through Band Alignment Engineering. Energy Environ. Sci. 2015, 8, 2928-2934.

(33) Meloni, S.; Moehl, T.; Tress, W.; Franckevičius, M.; Saliba, M.; Lee, Y. H.; Gao, P.; Nazeeruddin, M. K.; Zakeeruddin, S. M.;
Rothlisberger, U. Ionic Polarization-Induced Current-voltage Hysteresis in $\mathrm{CH}_{3} \mathrm{NH}_{3} \mathrm{PbX}_{3}$ Perovskite Solar Cells. Nat. Commun. 2016, 7, 10334.

(34) Pockett, A.; Eperon, G.; Sakai, N.; Snaith, H.; Peter, L. M.; Cameron, P. J. Microseconds, Milliseconds and Seconds: Deconvoluting the Dynamic Behaviour of Planar Perovskite Solar Cells. Phys. Chem. Chem. Phys. 2017, 19, 5959-5970.

(35) Pazoki, M.; Jacobsson, T. J.; Kullgren, J.; Johansson, E. M. J.; Hagfeldt, A.; Boschloo, G.; Edvinsson, T. Photoinduced Stark Effects and Mechanism of Ion Displacement in Perovskite Solar Cell Materials. ACS Nano 2017, 11, 2823-2834.

(36) Milot, R. L.; Eperon, G. E.; Snaith, H. J.; Johnston, M. B.; Herz, L. M. Temperature-Dependent Charge-Carrier Dynamics in $\mathrm{CH}_{3} \mathrm{NH}_{3} \mathrm{PbI}_{3}$ Perovskite Thin Films. Adv. Funct. Mater. 2015, 25, $6218-6227$.

(37) Wright, A. D.; Verdi, C.; Milot, R. L.; Eperon, G. E.; PérezOsorio, M. A.; Snaith, H. J.; Giustino, F.; Johnston, M. B.; Herz, L. M. Electron-phonon Coupling in Hybrid Lead Halide Perovskites. Nat. Commun. 2016, 7, 11775.

(38) Jacobsson, T. J.; Tress, W.; Correa-Baena, J. P.; Edvinsson, T.; Hagfeldt, A. Room Temperature as a Goldilocks Environment for $\mathrm{CH}_{3} \mathrm{NH}_{3} \mathrm{PbI}_{3}$ Perovskite Solar Cells: The Importance of Temperature on Device Performance. J. Phys. Chem. C 2016, 120, 11382-11393.

(39) Kawai, H.; Giorgi, G.; Marini, A.; Yamashita, K. The Mechanism of Slow Hot-Hole Cooling in Lead-Iodide Perovskite: First-Principles Calculation on Carrier Lifetime from Electron-Phonon Interaction. Nano Lett. 2015, 15, 3103-3108.

(40) Yang, Y.; Ostrowski, D. P.; France, R. M.; Zhu, K.; van de Lagemaat, J.; Luther, J. M.; Beard, M. C. Observation of a Hot-Phonon Bottleneck in Lead-Iodide Perovskites. Nat. Photonics 2015, 10, 5359.

(41) Hoke, E. T.; Slotcavage, D. J.; Dohner, E. R.; Bowring, A. R.; Karunadasa, H. I.; McGehee, M. D. Reversible Photo-Induced Trap Formation in Mixed-Halide Hybrid Perovskites for Photovoltaics. Chem. Sci. 2015, 6, 613-617.

(42) Pazoki, M.; Wolf, M. J.; Edvinsson, T.; Kullgren, J. Vacancy Dipole Interactions and the Correlation with Monovalent Cation Dependent Ion Movement in Lead Halide Perovskite Solar Cell Materials. Nano Energy 2017, 38, 537-543.

(43) Zhao, Y.-C.; Zhou, W.-K.; Zhou, X.; Liu, K.-H.; Yu, D.-P.; Zhao, Q. Quantification of Light-Enhanced Ionic Transport in Lead Iodide Perovskite Thin Films and Its Solar Cell Applications. Light: Sci. Appl. 2017, 6, e16243. 